\title{
Research Paper \\ Online Game Addiction Relationship With Cognitive Distortion, Parenting Style, and Narcissistic Personality Traits in Students
}

\author{
*Arash Zandi Payam ${ }^{1}$ 이, Zeynab Mirzaeidoostan² $\mathbb{C}$
}

1. MSc., Lecturer, Department of Psychology, Payame Noor University, Tehran, Iran

2. MSc., Department of Psychology, Faculty of Education \& Psychology, Shahid Chamran University of Ahvaz, Ahvaz, Iran.

\begin{tabular}{|l|l|l}
$\begin{array}{c}\text { Use vourdevice to scan } \\
\text { and read the article online }\end{array}$ & $\begin{array}{l}\text { Cftation Zandi Payam A, Mirzaeidoostan Z. [Online Game Addiction Relationship With Cognitive Distortion, Parenting Style, } \\
\text { and Narcissistic Personality Traits in Students (Persian)]. Iranian Journal of Psychiatry and Clinical Psychology. 2019; 25(1):72- } \\
\text { 83. http://dx.doi.org/10.32598/ijpcp.25.1.72 }\end{array}$ \\
dol http://dx.doi.org/10.32598/ijpcp.25.1.72
\end{tabular}

Received: 05 Jul 2018

Accepted: 09 Oct 2018

Available Online: 01 Apr 2019

Keywords:

Video game, Cognitive distortion, Child-rearing, Narcissism

\begin{abstract}
AB STRACT
Objectives Online games in recent years have become one of the most popular hobbies among teenagers due to their diverse and attractive features. The purpose of this study was to investigate the role of cognitive distortion, parenting style, and narcissistic personality traits in online games in high school boy in Sahneh City, Iran.

Methods This is a survey and a correlational study. The study sample consists of 200 male high school students in the city of Sahneh, Iran who were selected randomly by a multi-stage sampling method. For data collection, online gaming addiction questionnaire, cognitive disturbance questionnaire, parenting styles questionnaire, and narcissistic personality traits questionnaire were used. In this research, the correlation and multiple regression were used to analyze the obtained data in SPSS V. 21.

Results The findings showed that online addiction can be predicted by a linear combination of predictive variables. Based on regression analysis, parenting style $(P \leq 0.001, \beta=0.46)$, narcissistic personality traits $(P \leq 0.001, \beta=0.34)$, and cognitive disturbance $(P \leq 0.025, \beta=0.11)$ could significantly predict students' addiction to online games.

Conclusion These variables can play an effective role in addiction to play online games. Considering these variables, authorities of schools and other educational institutions should design and implement appropriate monitoring and control programs for this common problem.
\end{abstract}

\section{Extended Abstract}

\section{Introduction}

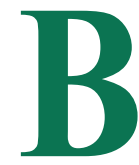

ecause of their diverse and attractive features, online games have become one of the most popular hobbies among teenagers in recent years. The online game addiction is defined as "excessive and compulsory use of video games that lead to social and emotional problems, and the user, despite the problems, is not able to control this excessive use"
[1]. The World Health Organization also announced that online game addiction should be recognized as a disease, as experts agree on the risk of addiction to these games. Although this is said to be due to the widespread expansion of online games, it is more common among young people. Mr. Yasarovich said, "it is still too early to predict the extent of the problem". Online game addiction is a relatively new concept and there is still not enough information about the disease and its dissemination factors at the population level [2].

* Corresponding Author:

Arash Zandi Payam, MSc.

Address: Department of Psychology, Payame Noor University, Tehran, Iran.

Tel: +98 (918) 3123449

E-mail: zandipayam.arash@gmail.com 
Research shows that introvert people were more addicted to online games. They were more worried about their identity and more insecure about peers [5]. These concerns about themselves and others, along with their characteristics can lead these teenagers to avoid real social activities [6]. They may also try to cover their social needs through online games, where they can interact with others remotely and in a superficial manner [7]. The negative consequences of online addiction can be classified into three broad categories: physical problems (fatigue, physical pain, reduced sleep time, eating disorder), personal life problems (conflicts with friends or family, low social participation), and professional failures (loss of work or school, poor performance) [3]. Some psychologists report the link between depression, anxiety, and symptoms of attention deficithyperactivity disorder with online addiction $[8,9]$.

Cognitive distortion structures, introduced by Aaron, T Beck and Albert Ellis, are generally defined as false arguments that play an important role in the development of many mental disorders. According to the cognitive-behavioral model, cognitive distortion plays an important role in Internet addiction [12]. Parenting style is defined as the various, normal, and natural behaviors that are used by parents to control and socialize with their children. The results of the research showed that parents of people with Internet addiction do not have the warmth and intimacy, and their children consider their parents (especially their mothers) to be punitive and exclusionary [13].

Narcissistic personality is a type of personality characterized by features such as extreme fantasies about power, beauty and success, having a great deal of sensitivity to criticism, and unparalleled feelings. In fact, high narcissistic personality traits are associated with an increase in online game addiction [17]. The purpose of this study was to investigate the role of cognitive distortion, parenting style, and narcissistic personality traits in online games addiction in high school boy students in Sahneh city, Iran. This research examines two hypotheses: cognitive distortion, parenting style and narcissistic personality traits have a meaningful relationship with addiction to online games in students; cognitive distortion, parenting style and narcissistic personality traits predict addiction to online games in students. .

\section{Method}

The research method is descriptive correlational. The statistical population included all high school students (second period) in the academic year of 2016-2017 in the city of Sahneh. Using a multistage random sampling method, a total of 200 male students were selected from the statistical population. For data collection, online gaming addiction questionnaire (Persian version of online addiction test, translated and Standardize by Zandi Payam et al. [18]), cognitive distortion questionnaire (Abdollah Zadeh and Salar cognitive distortion questionnaire), parenting styles questionnaire (Baarmind), and narcissistic personality traits questionnaire (the shortened version of a 40-item narcissistic character questionnaire designed by Ames, et al. [23]) were used. In this research, the collected data were analyzed by descriptive statistics such as mean, standard deviation, and inferential statistics like correlation matrix and multivariate regression in SPSS V. 21.

\section{Results}

The results of the significant tests of the complete model of the correlation analysis revealed a significant relationship between online addiction variables and cognitive distortion, parenting styles, and narcissistic personality traits. The findings showed that online addiction can be predicted by a linear combination of predictive variables. According to Table 1, the regression analyses indicate that the parenting style $(\mathrm{P} \leq 0.001$, $\beta=0.46)$, narcissistic personality traits $(\mathrm{P} \leq 0.001, \beta=0.34)$, and cognitive distortion $(\mathrm{P} \leq 0.225, \beta=0.11)$ can significantly predict student addiction to online games.

\section{Discussion}

According to our study results, the level of narcissism and cognitive distortion is high in addicted online gamers, and the parents of addicted people have more authoritarian childrearing style. Student addiction can lead to a drop in academic performance. Of course, in this context, the parental style plays a significant role. It seems that holding meetings at schools with the presence of parents, teachers, and psychologists to identify parenting styles, provide guidance on how parents interact

Table 1. The results of the multiple regression analysis of prediction of addiction to online games

\begin{tabular}{ccccccc}
\hline Predictors & $\boldsymbol{\beta}$ & $\mathbf{t}$ & $\mathbf{P}$ & $\mathbf{R}$ & $\mathbf{R}^{2}$ & $\mathbf{F}$ \\
\hline Cognitive distortion & 0.11 & 2.25 & 0.025 & & & \\
Child-rearing style & 0.46 & 10.28 & 0.001 & 0.55 & 0.3 & 17.9 \\
Narcissism & 0.34 & 7.57 & 0.001 & & \\
\hline
\end{tabular}


and control their parenting type in dealing with children's online gaming. Therefore, the results of this study can be used by practitioners in the field of National Computer Games and the Ministry of Sports and Youth in the area of children's and adolescent's health family counseling and psychotherapy. Detecting the static and dynamic variables in the process of addiction to the games helps professionals focus on these factors and organize their activities, which will result in providing more effective services to the clients.

\section{Ethical Considerations}

Compliance with ethical guidelines

The participants signed the informed consent form and had the right to leave the study at any time.

Funding

This research did not receive any specific grant from funding agencies in the public, commercial or not-for-profit sectors.

Authors contributions

Conceptualization: All authors; Methodology: Arash Zandipayam; Validation: All authors; Analysis: All authors; Investigation: Arash Zandipayam; Sources: Arash Zandipayam; Preparation, writing-review \& editing: Arash Zandipayam; and Project administration: All authors.

\section{Conflict of interest}

The authors declared no conflict of interest. 


\title{
ييشبينى اعتياد به بازى آنلاين با توجه به تحريف شناختى، سبك فرزنديرورى و ويزًّى شخصيتى خودشيفته در دانش آموزان
}

\author{
• آرش زندى ييام' هـ زينب ميرزايىدوستان' \\ 1- كارشناس ارشده مربى، كروه روانشناسى بالينى، دانشعاه هييام نور، تهران، ايران.

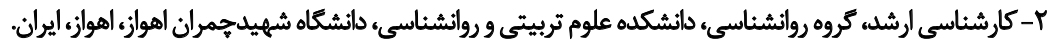

\begin{abstract}
حكيد

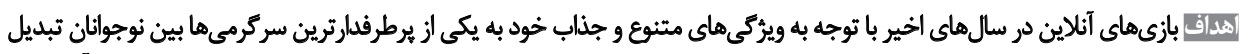

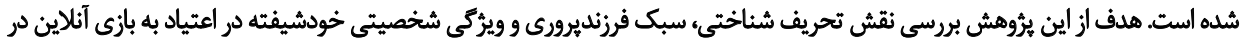

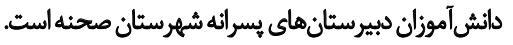

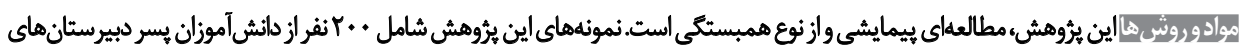

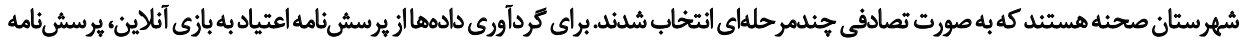

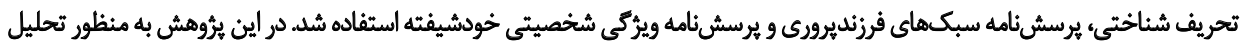

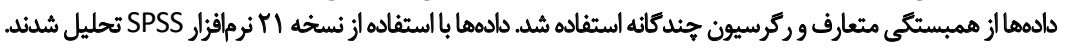

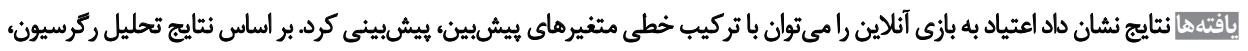

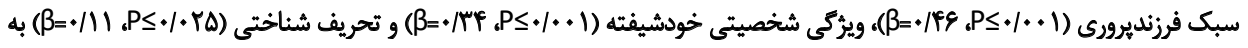

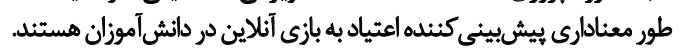

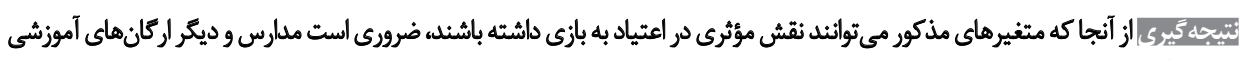

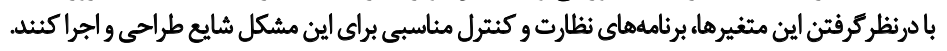

تاريخ دريافت: If

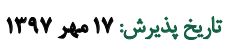

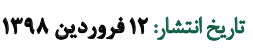

كليدوازوها:

بازى ويدئويى، تحريف

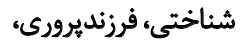
خودشيفتكى فرزني

عبارت است ازئ الكوى رفتارى مربوط به بازىهاى ديجيتال يا

مقدمه

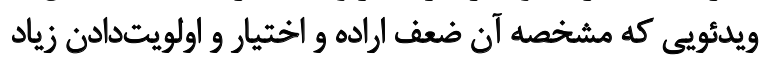

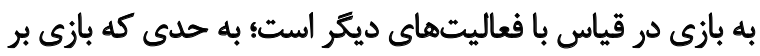
ديكر علايق فرد مقدم باشد.

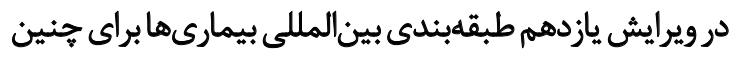

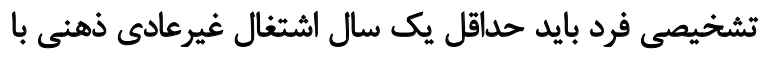

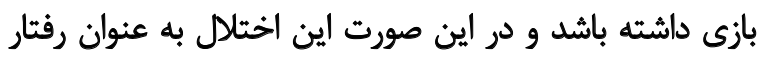

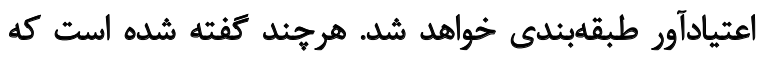

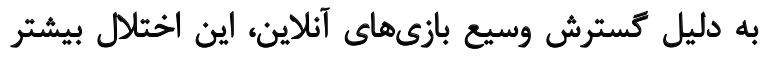

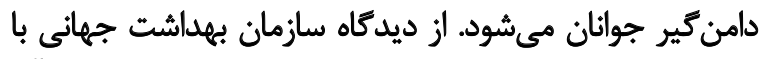

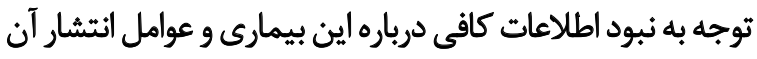

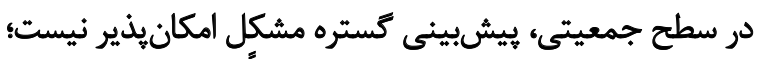

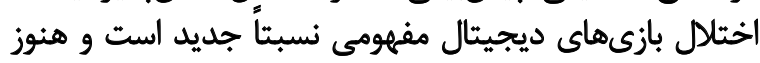

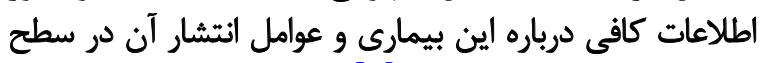

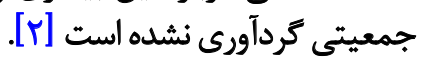

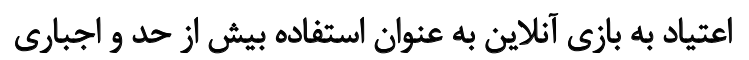

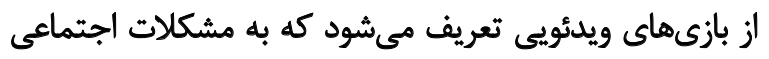

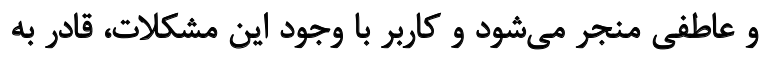

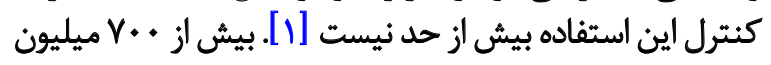

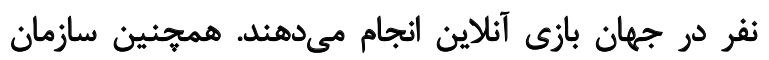

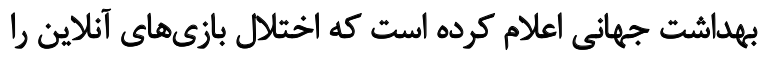

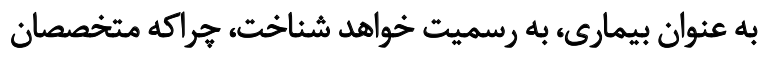

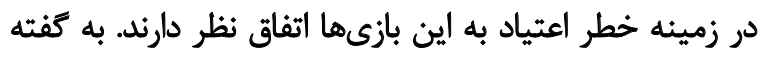

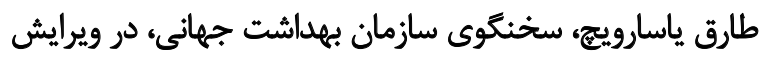

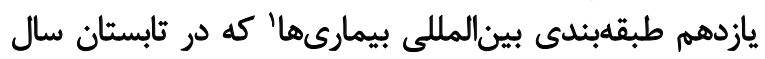

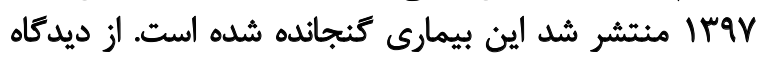

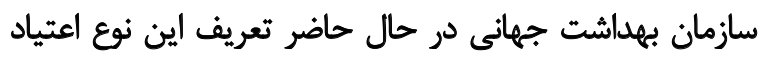

1. International Classification of Diseases-XI (ICD-11)

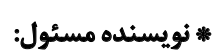

$$
\begin{aligned}
& \text { آرش زندى ثياميام } \\
& \text { نشاني: تهران، دانشعاه ئيام نور، كروه روانشناسي بالينى. } \\
& \text { تلفن: } \\
& \text { يست الكترونيكي: zandipayam.arash@gmail.com }
\end{aligned}
$$




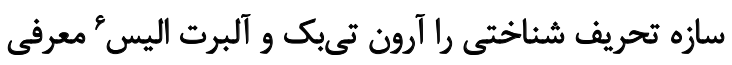

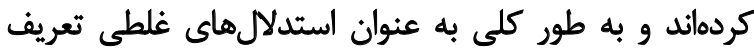

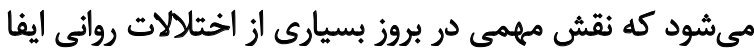

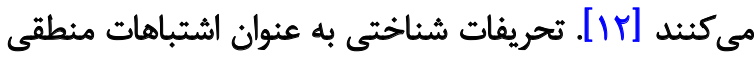

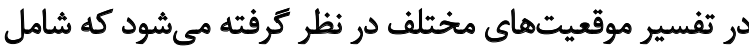

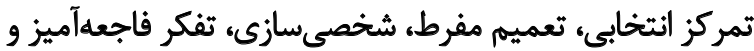

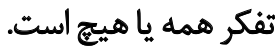

نقش مهم تحريفات شناختى در توسعه جدى اختلالاتى مائند

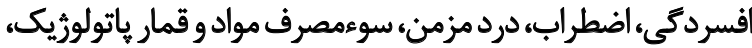

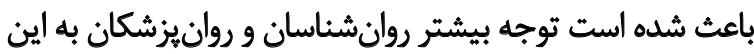

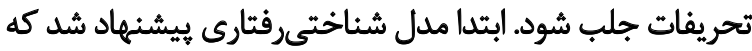

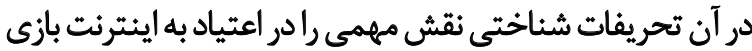

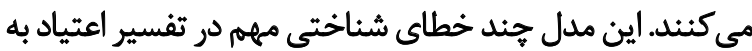

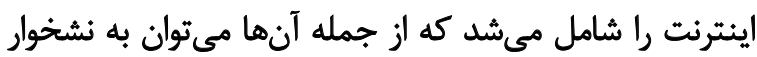

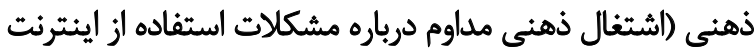

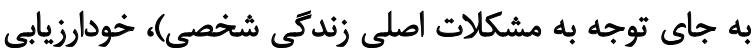

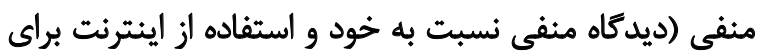

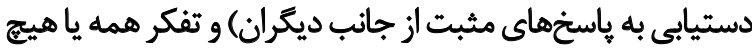

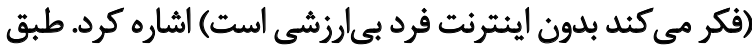

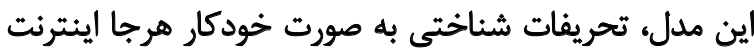

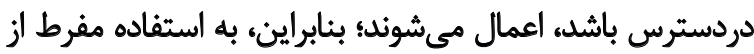

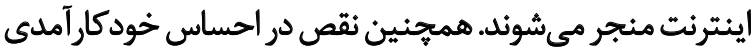

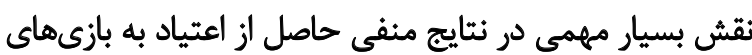

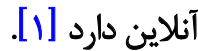

نوع برخورد والدين در قالب رفتارهاى متنوع، بهنجار و

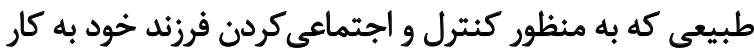

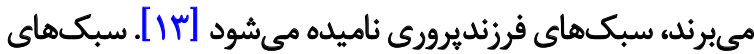

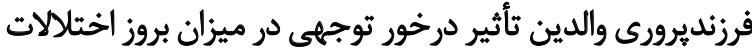

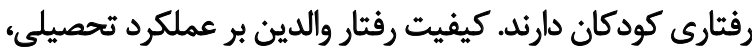

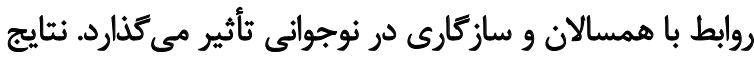

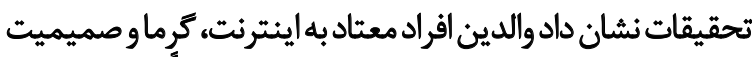

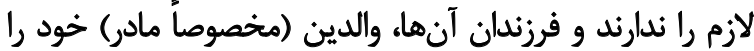

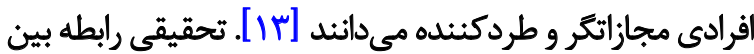

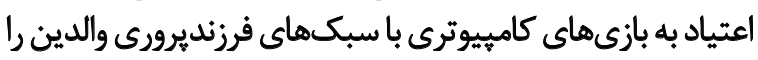

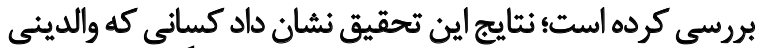

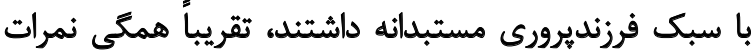

بالايى در اعتياد به بازى داشتند [ [ أ]

همجنين نتايج تحقيقات نشان داد والدين با سبكهاي

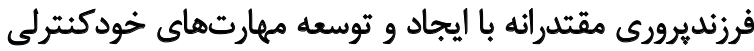

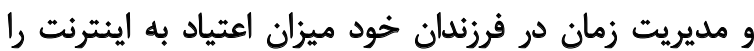

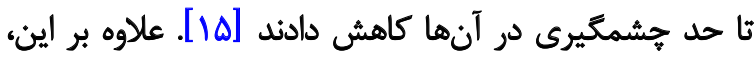

6. Aaron Temkin Beck \& Albert Ellis

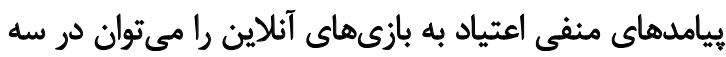

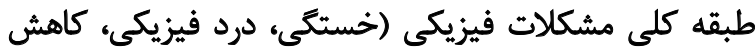

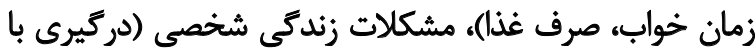

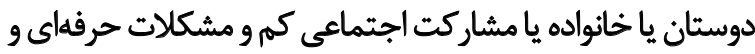

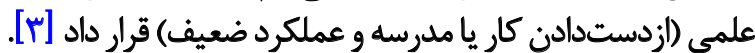

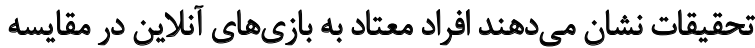

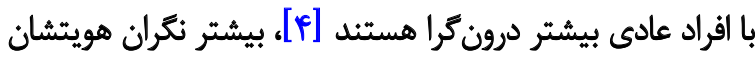

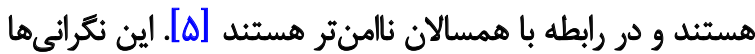

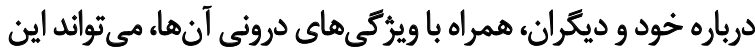

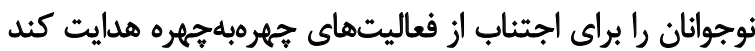

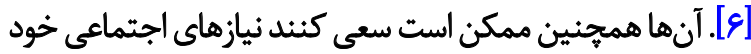

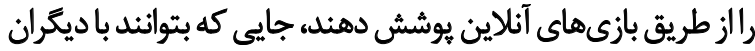

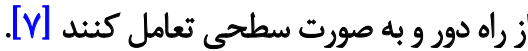

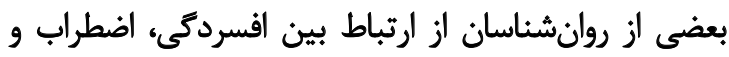

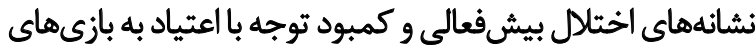

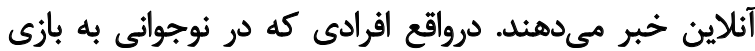

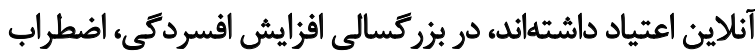

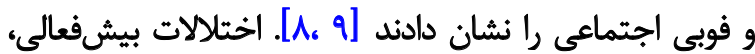

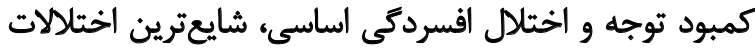

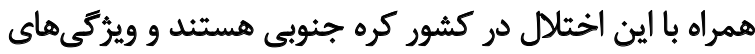

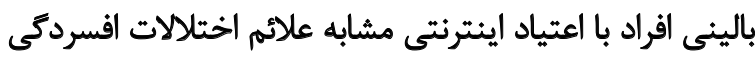

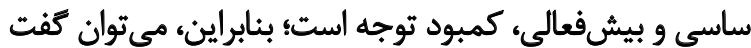

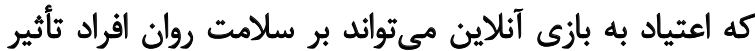

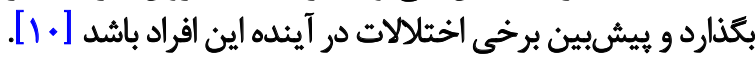

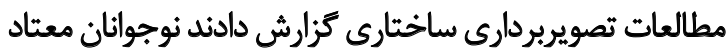

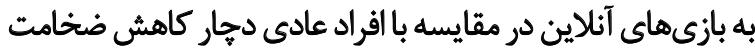

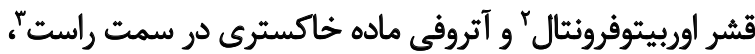

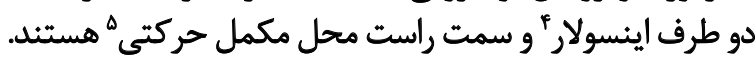

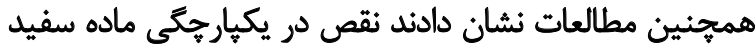

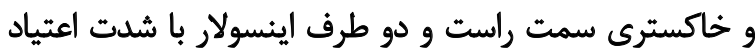

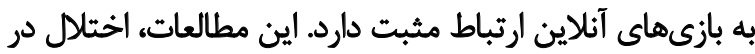

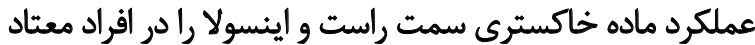

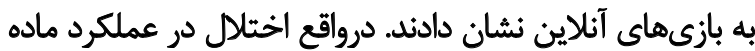

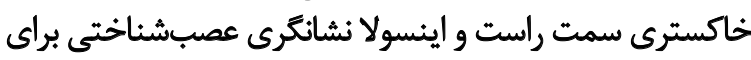

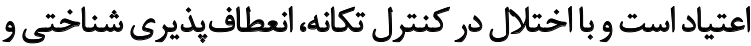

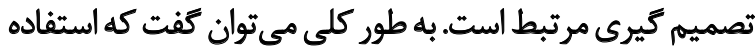

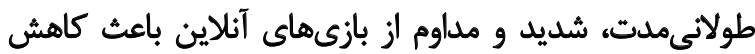

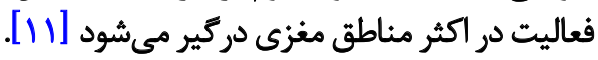

2. Orbitofrontal cortex

3. Right Orbital Frontal Cortex (R-OFC)

4. Bilateral insula

5. Right supplementary motor area 


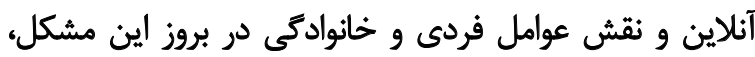

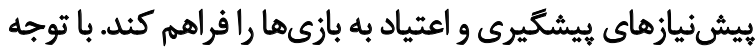

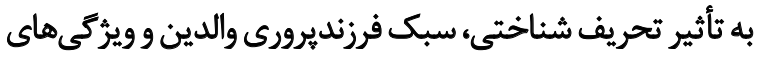

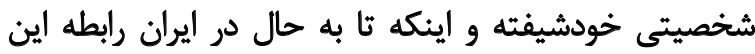

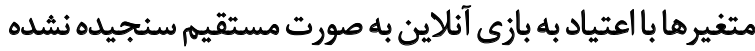

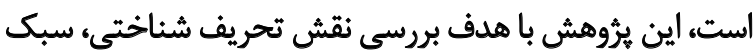

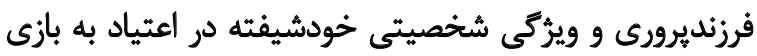

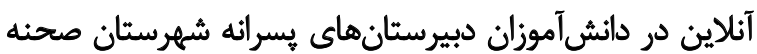

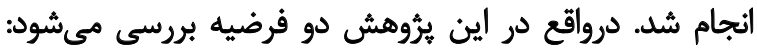

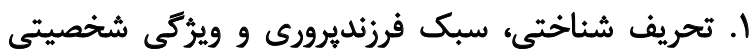

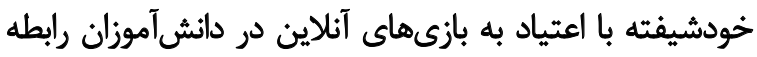

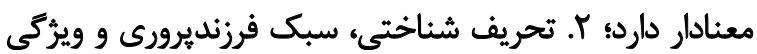

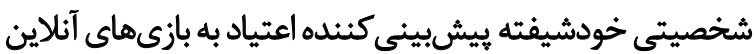
در دانش آموزان هستيند.

(2)

روش اين يُروهش توصيفى و از نوع همبستخى است. جامعه

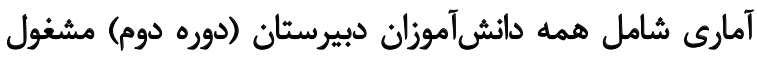

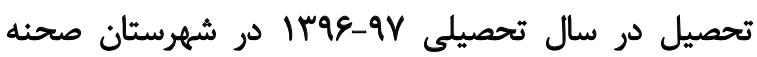

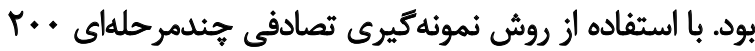

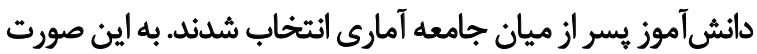

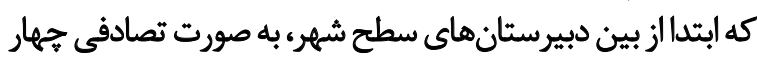

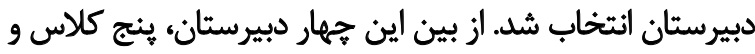

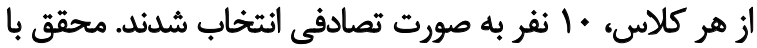

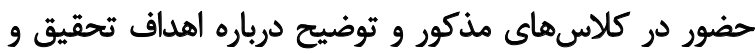

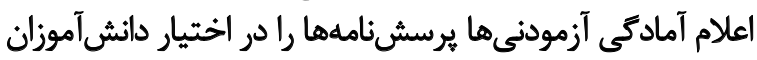

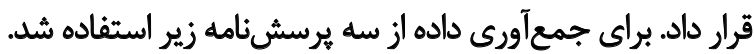

\section{ير سشئامه اعثياد به بازئهاي آثلاين}

در اين يُروهش نسخه فارسى مقياس اعتياد به بازى آنلاين كه

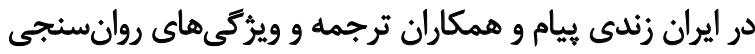

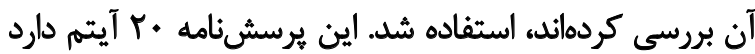

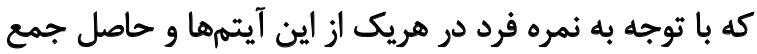

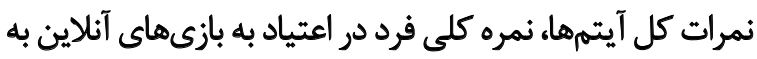

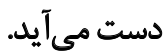

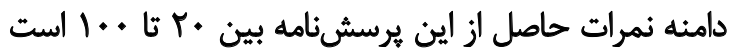

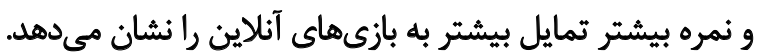

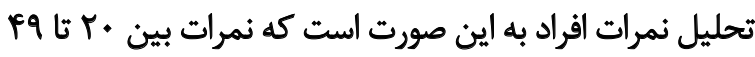

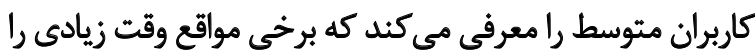

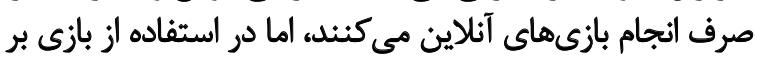

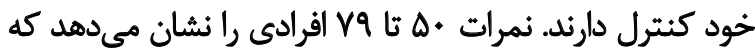

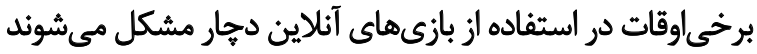

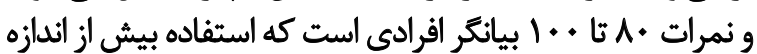

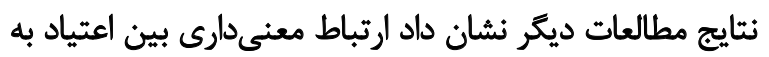

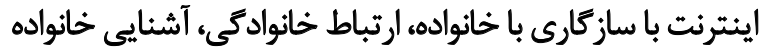

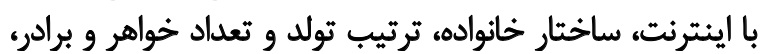

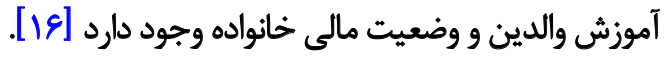

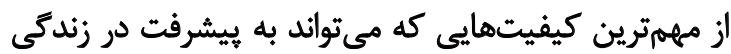

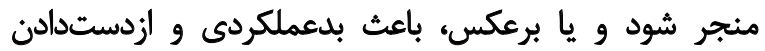

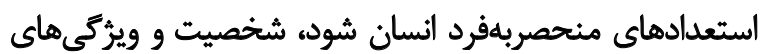

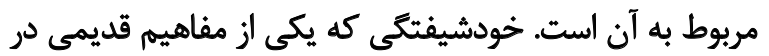

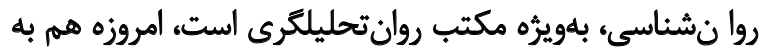

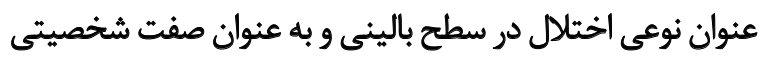

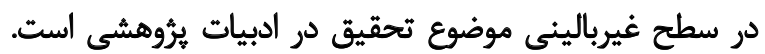

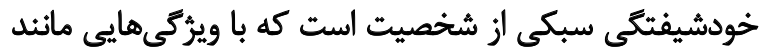

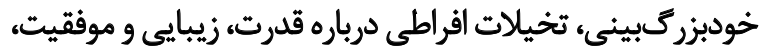

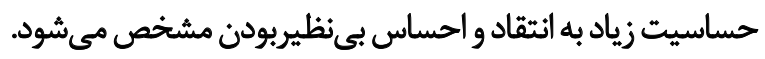
يافتهاى بروهشى در جمعيتهاى بهنجار نشان مىدهد

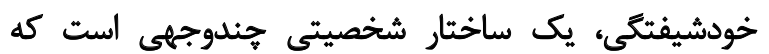

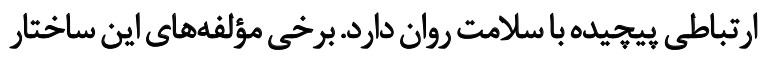

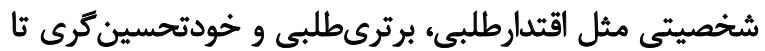

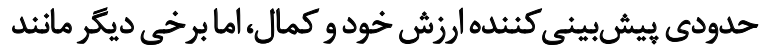

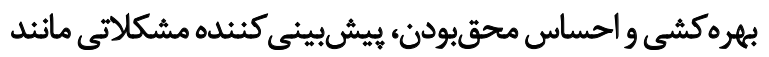

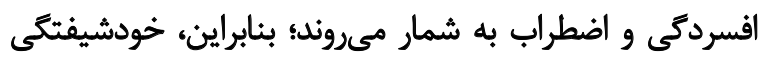

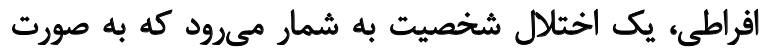

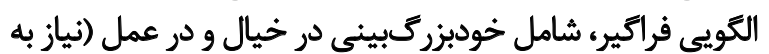

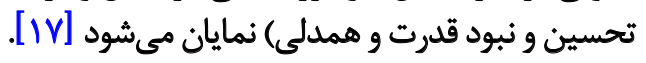

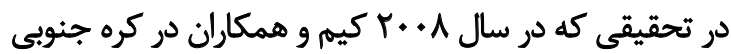

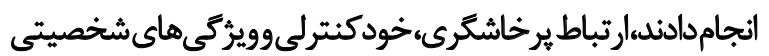

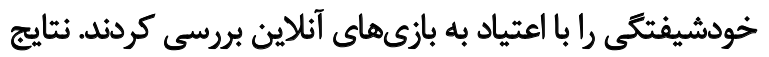

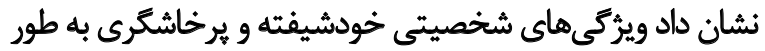

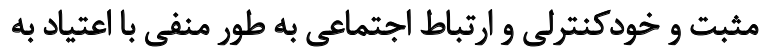

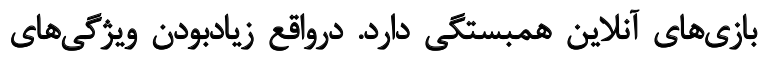

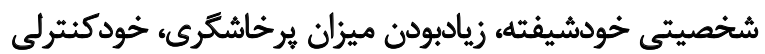
ضعيف، ارتباط ميانفردى ضعيف و بيكارى بادي إفزايش ميزان اعتياد به بازىهاى آنلاين مرتبط است [. [1]. نوجوانبودن قشر عظيمى از جمعيت كشور، ضرورت توجانه

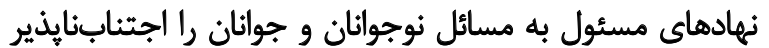

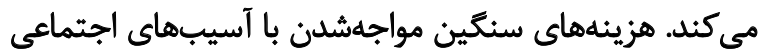

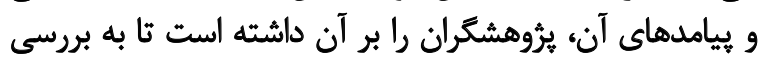

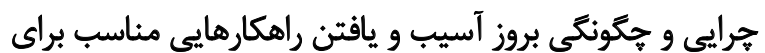

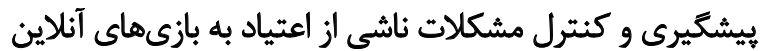
بيردازند.

يافتههاى اين بثروهش به اين دليل اهميت دارد كه مى بواند

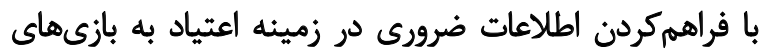


آلفاى بهدست آمده از اين يرسش آنامه به صورت استاندارد برابر با

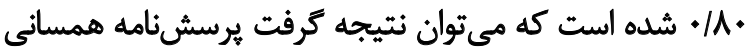

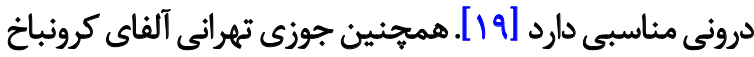

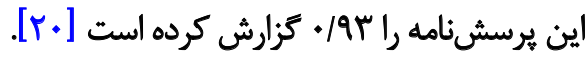

\section{سبك فوزنديوروى}

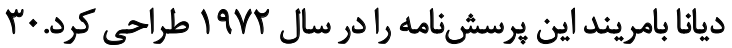

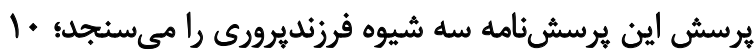

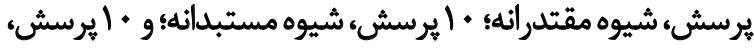

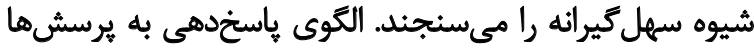

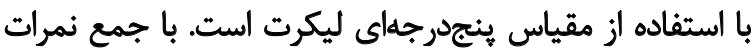

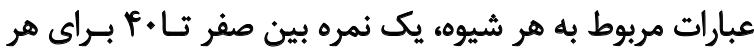

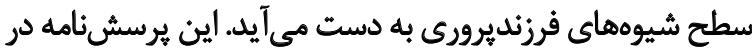

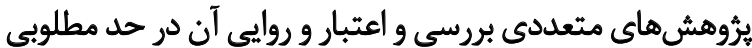

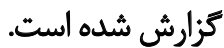

در يكى از تحقيقات با استفاده از روش بازآزمايى، هايايى اين

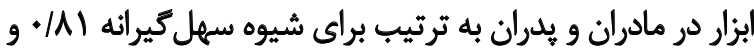

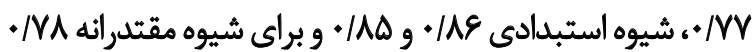

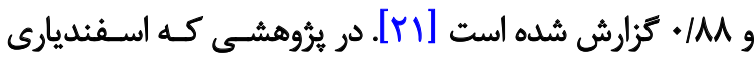

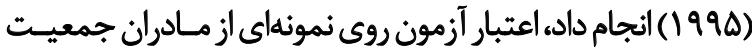

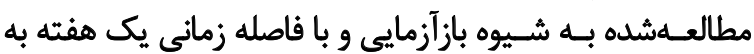

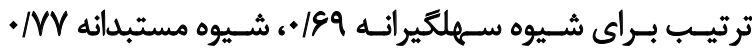

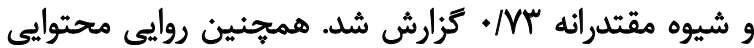

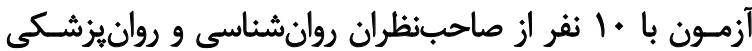

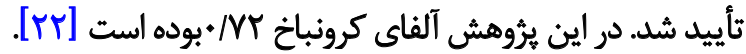

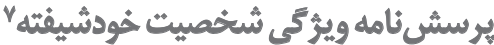

نسخه كوتاهشده هرسش نامه · ·fكويهاي شخصيت خودشيفته

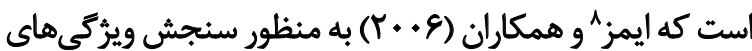

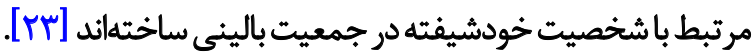

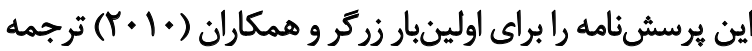

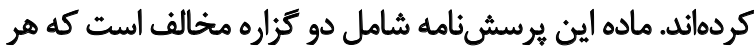

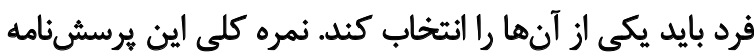

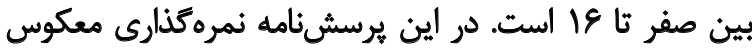

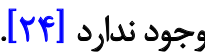

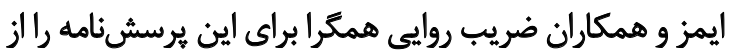

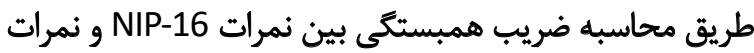

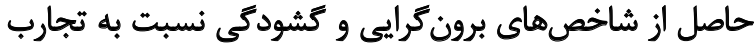

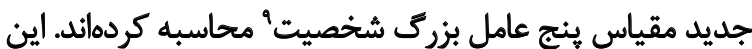

7. Narcissistic Personality Inventory (NIP-16)

8. Ames

9. Big Five Inventory (BFI)
ز بازىهاى آنلاين مشكلات جدى را در زندكى آنان ايجاد كرده

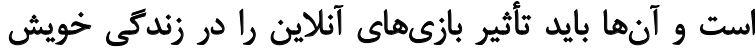

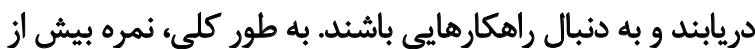

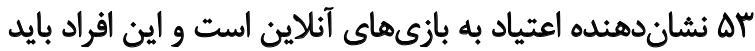

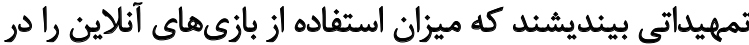
خود كنترل كثند.

هريك از سؤالات با مقياس ينجرتبهاى ليكرت نمرهكذارى

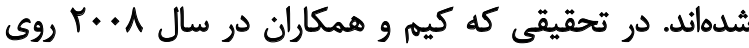

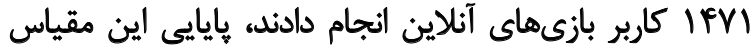

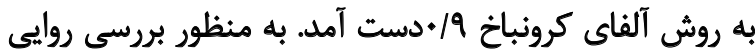

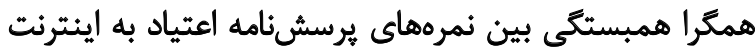

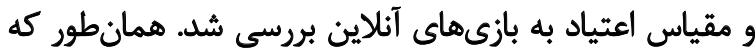

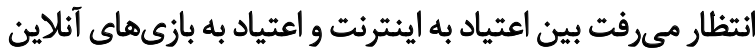

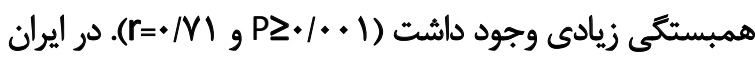

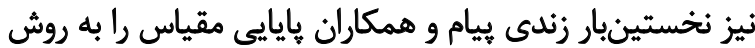

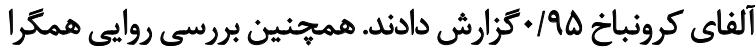

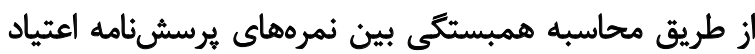

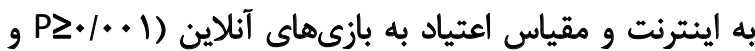

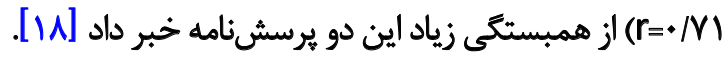

\section{تحريف شناختى}

در اين تحقيق از يرسشنامه تحريفهاى شناختى عبدالهزاده

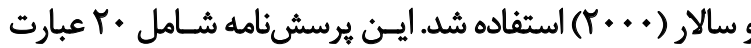

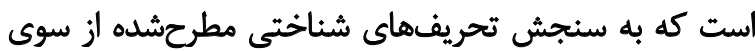

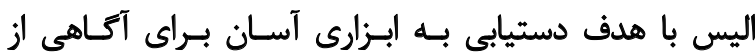

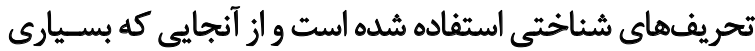

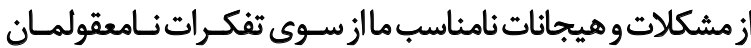

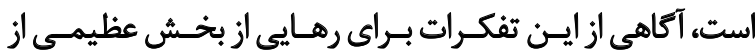

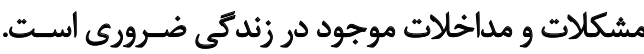

عبارتهاى اين يرسش نامه عبارتند از: شماره ا و r: تفكر همه

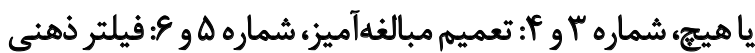

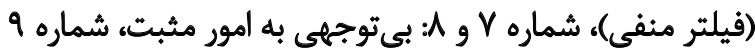

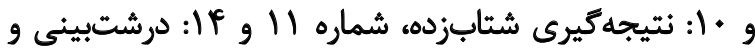

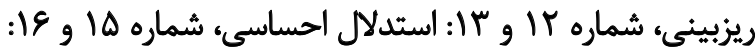

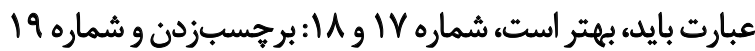

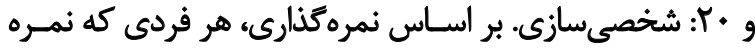

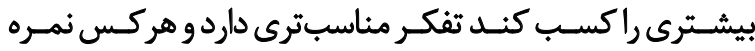

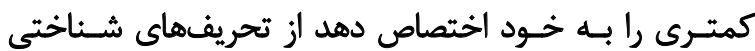

$$
\text { بيشـترى اسـتفاده كـرده است. }
$$

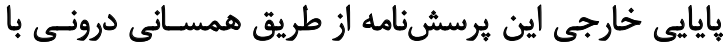

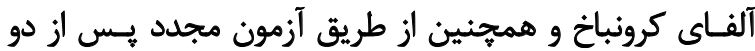

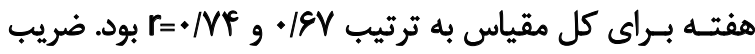




\section{معناداري مدل كامل همبستئى متعارف}

آزمونهاى معنادارى مدل كامل تحليل همبستئى متعارف

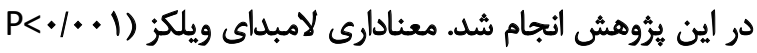

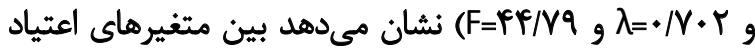

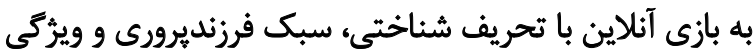

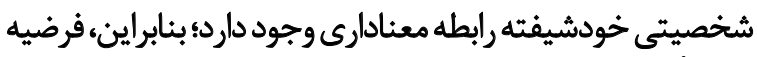

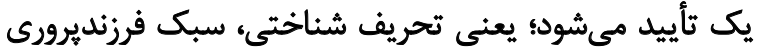

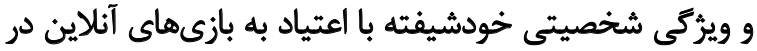

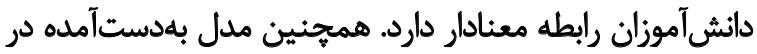

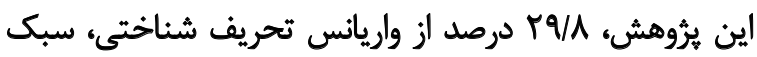

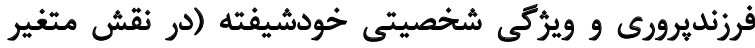

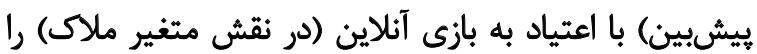

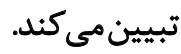

براى بررسى فرضيه دوم، قدرت يّيشبينى اعتياد به بازى في

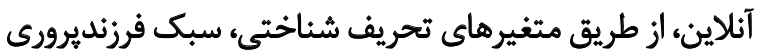

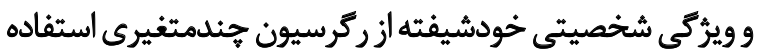

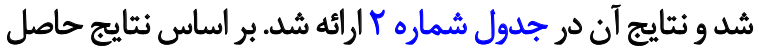

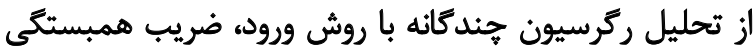

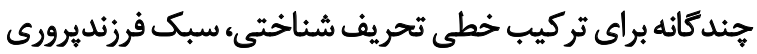

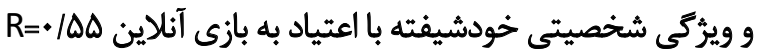

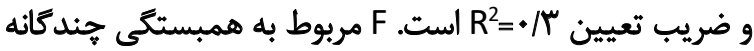

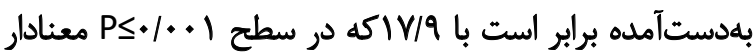

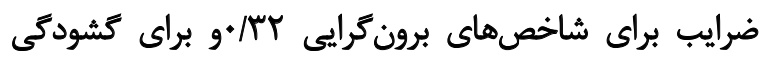

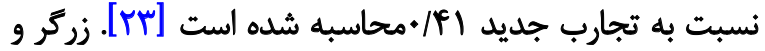

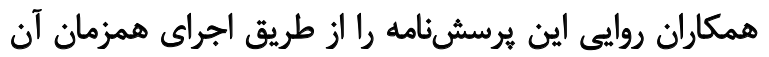

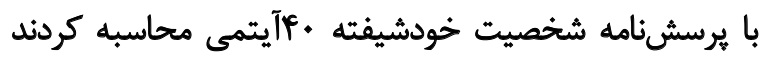

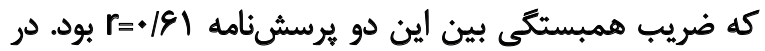

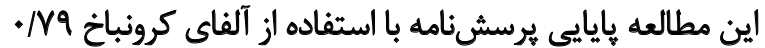

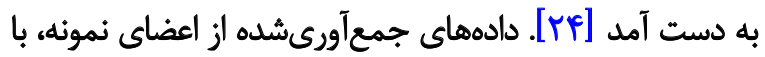

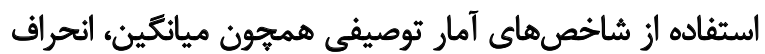

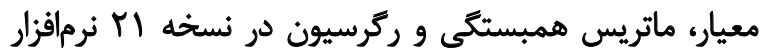
SPSS

يافتهاه

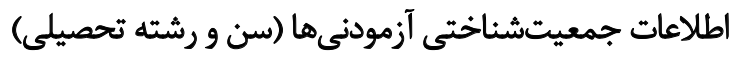

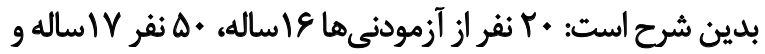

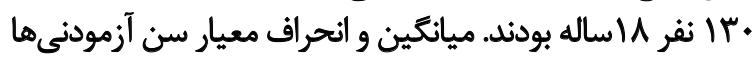

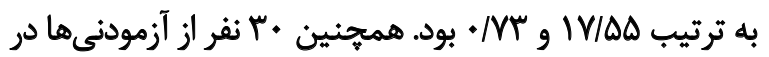

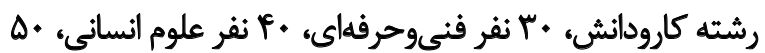

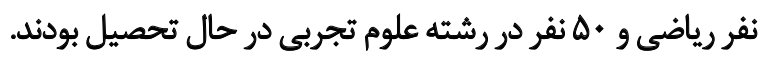
در جدول شماره ا شاخصهاى متغيرهاى بيشبين و ملاك

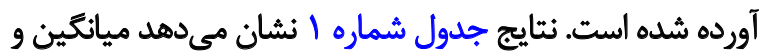

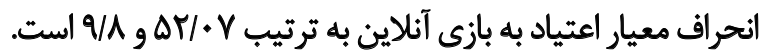

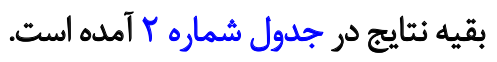

جدول ا. ميانتين و انحراف معيار متغيرها

\begin{tabular}{|c|c|c|}
\hline ميانكين土|نحراف معيار & تعداد & متغير \\
\hline$\Delta r / \cdot V \pm q / \Lambda$ & $r+\cdot$ & اعتياد به بازى هاى آنلاين \\
\hline$|Q| \cdot r \pm m / \Lambda$ & Y.. & تحريف شناختى \\
\hline$\Gamma / T \pm T / \Lambda$ & $r+$. & سبك استبلادي \\
\hline $\mid f / \Lambda \pm \Delta / \Lambda$ & $r \cdot$. & سبك مقترائه \\
\hline $\mid \& / \Delta \pm \Delta / r$ & $r \cdot$. & سبك سهل كيرائه \\
\hline $1 \cdot / W \pm r / \Delta$ & $r+$. & خوششيفتكى \\
\hline
\end{tabular}

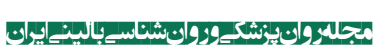

جدول r. نتايج حاصل از تحليل ركرسيون جندكانه بيشبينى اعتياد به بازىهاى آنلاين بر اساس تحريف شناختى، سبك فرزنديرورى و ويركى شخصيتى خودشيفته

\begin{tabular}{|c|c|c|c|c|c|c|}
\hline $\mathbf{P}$ & $\mathbf{T}$ & $\beta$ & F & $\mathbf{R}^{r}$ & $\mathbf{R}$ & مثغيرهاى ييشبين \\
\hline + + ro & T/TA &.$/ 11$ & & & & تحريف شناختى \\
\hline$* 1 . .1$ & $1 . / \mathrm{MA}$ &.$/ 48$ & IV/a & $\cdot / \mu$ & $\cdot / \Delta \Delta$ & سبك فرزنديرورى \\
\hline .1 .01 & $V / \Delta V$ &.$/ m$ & & & & ويرّكي شخصيتي خودشيفته \\
\hline
\end{tabular}


بر اين، نوجوانانى كه در جلسات رفتاردرمانى "ا شناختى شركت

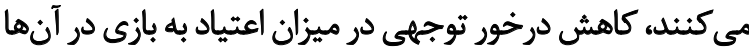

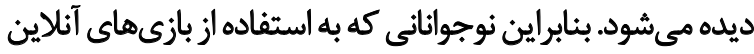

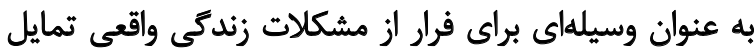

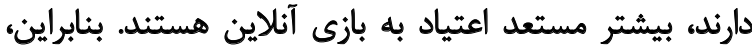

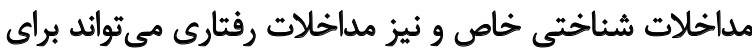

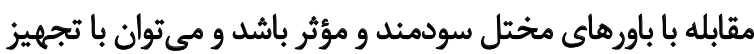

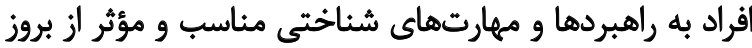

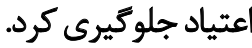
نتايج اين تحقيق حاكى از اين بود كه سبك فرزنديرورى والدين

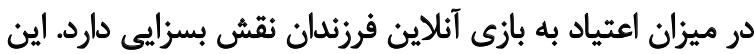

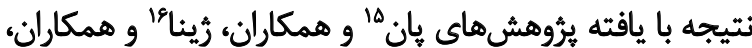

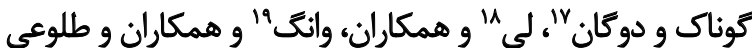

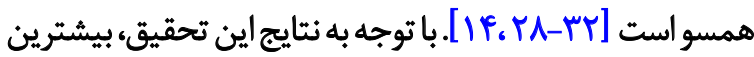

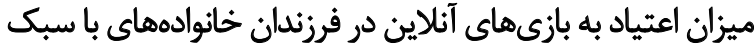

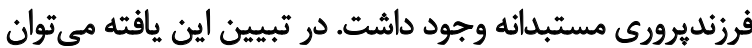

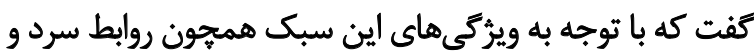

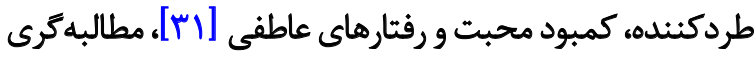

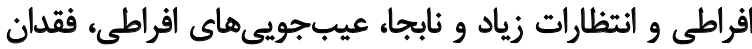

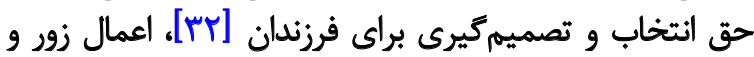

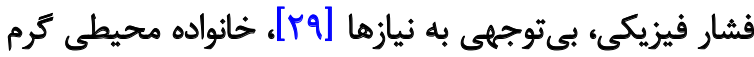

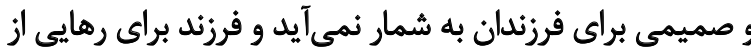

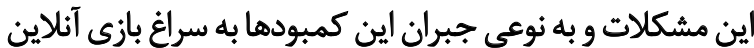

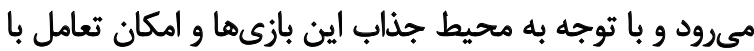
افراد مختلف علاقه او براى ادامه انجام بازى افزايش مي بـ يابداب والدينى كه سبكشان فرزنديرورى مقتدرانه است، با كرما و

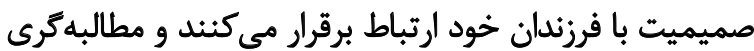

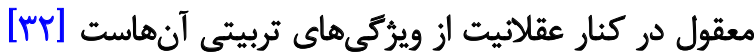

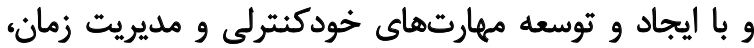

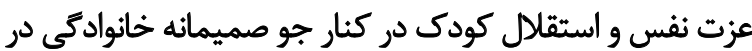

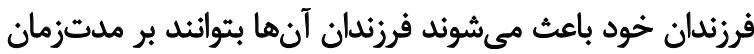

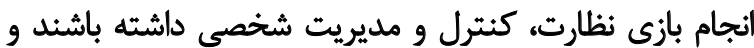

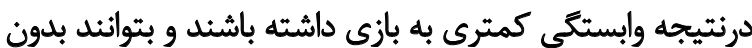

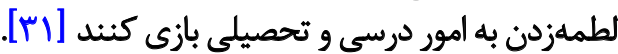

يكى ديكر از نتايج اين يُروهش رابطه معنادار بين ويرَّى هاي شخصيتى خودشيفته و اعتياد به بازىهائ آنلاين بود كه اين اين يناين

14. Cognitive Behavioral Therapy (CBT)

15. Pan

16. Xina

17. Gunuc \& Dogan

18. Li

19. Wang
با توجه به ضريب تعيين بلدستآمده، حدود • م درصد از بازي

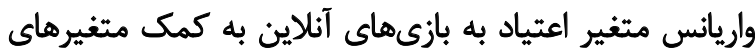

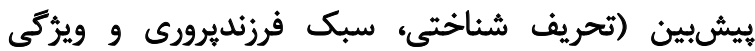

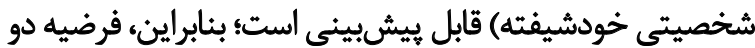

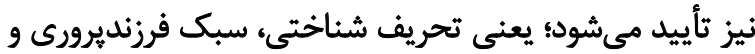

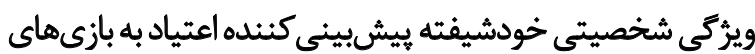

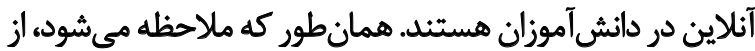

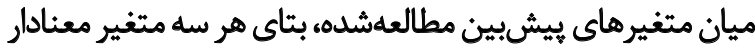

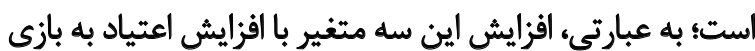

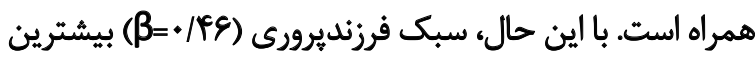

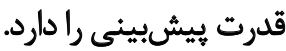

ثب

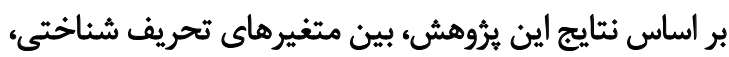

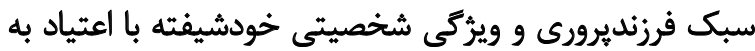

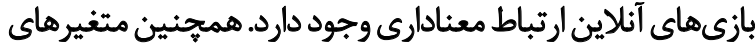

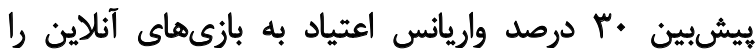

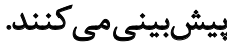

با توجه به نتايج اين يرؤوهش بين تحريف شناختى و اعتياد

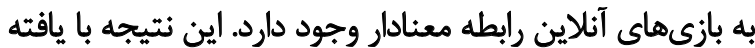

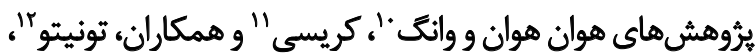

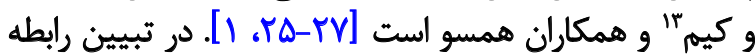
تحريف شناختى واعتيادبه بازى آنلاين مى توان كفت كنه

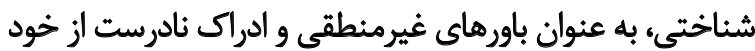

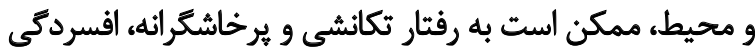

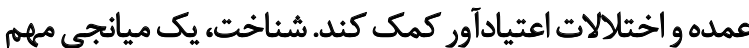

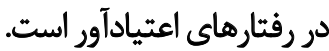

وجود تحريف شناختى باعث مختل شدن رفتار خودتنظيمى

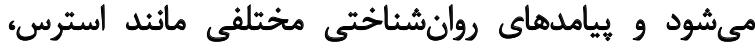

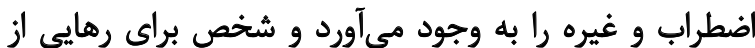

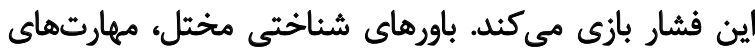

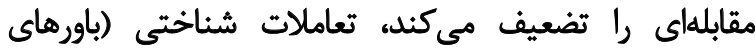

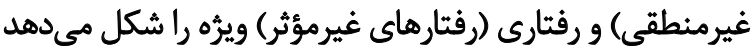

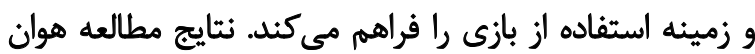

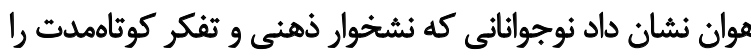

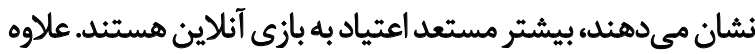

10. Huanhuan \& Wang

11. Kirisci

12. Toneatto

13. Kim 


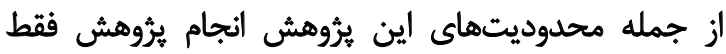

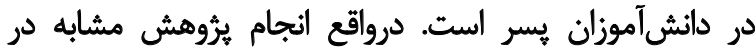

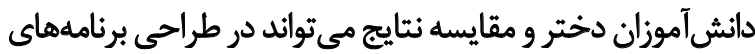

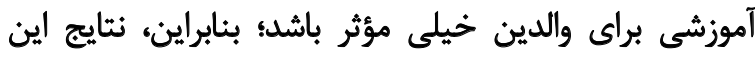

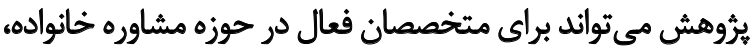

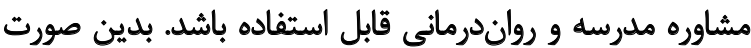

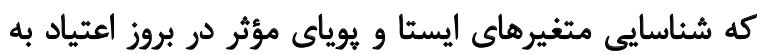

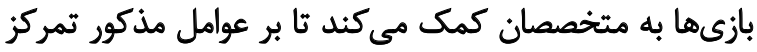

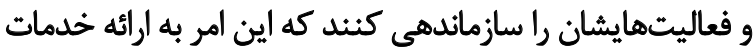
مؤثرتر به مراجعان منجر مي مانشود.

علاوه بر اين ارائه فراهمشدن اطلاعاتى در زمينه انغيزههاى

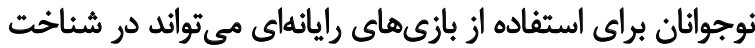

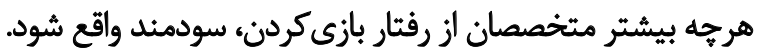

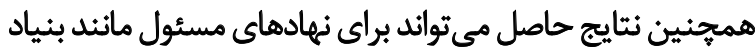

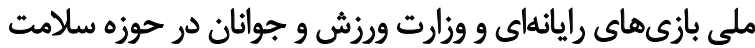

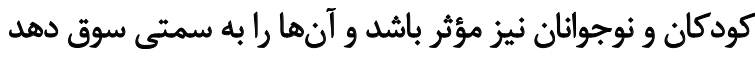

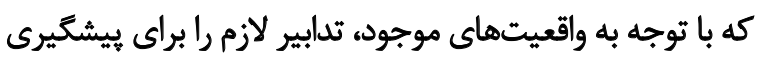

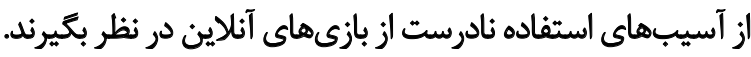

\section{ملاحظات اخلاقي \\ يبيروى أز اصول الخالاق يُؤهش}

همه اصول اخلافى در اين مقاله رعايت شده است.

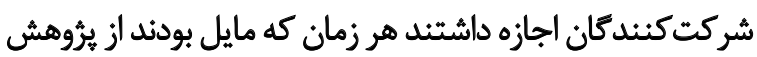

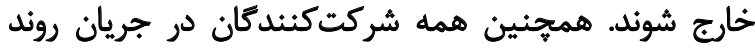

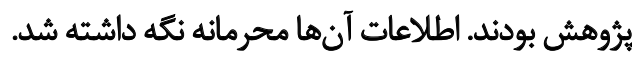

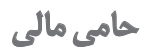

اين مقاله حامى مالى ندارد.

$$
\text { مشاركت نويسند }
$$

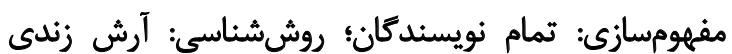

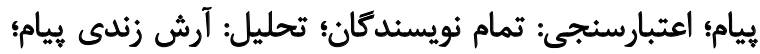

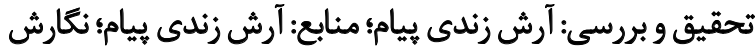

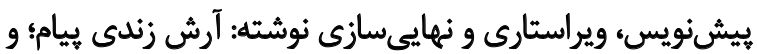
مديريت يروزه: تمام نويسندكان.

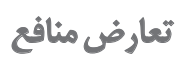

بنابر اظهار نويسندكان، اين مقاله تعارض منافع ندارد.

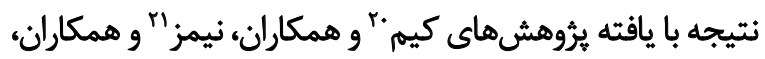

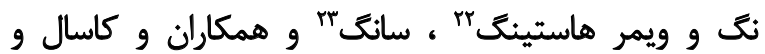

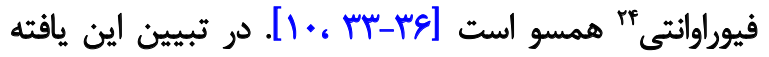

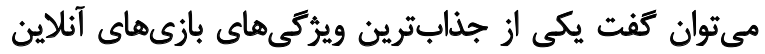

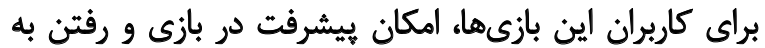

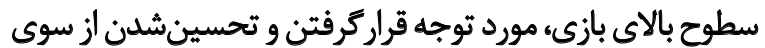

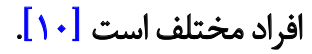

با توجه به إين مفهوم كه افراد با ويثزى هاى شخصيت

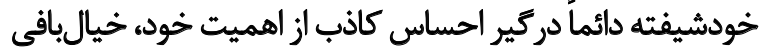

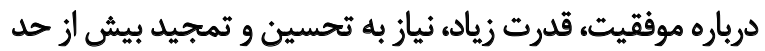

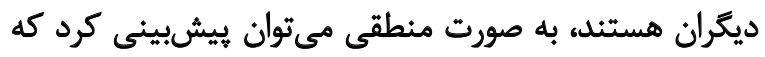

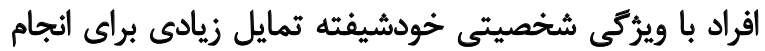

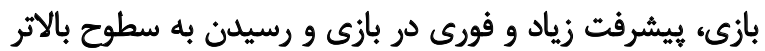

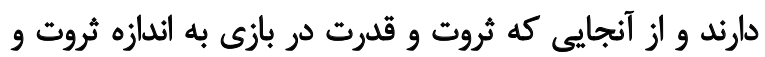

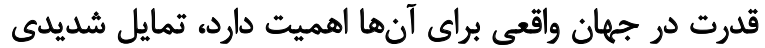

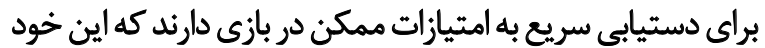

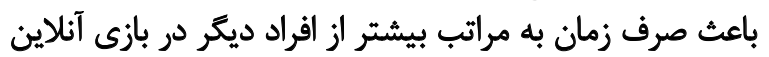

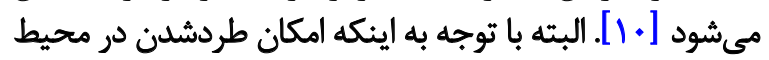

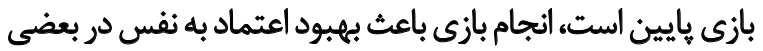

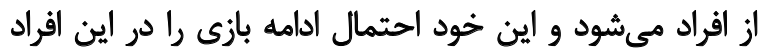

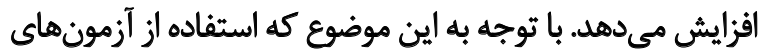

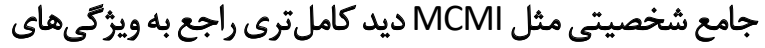

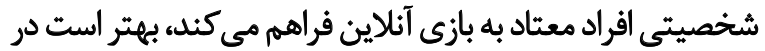

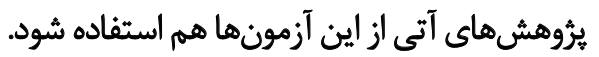

$$
\text { نتيجهيرى }
$$

به طور خلاصه طبق نتايج اين يُروهش، در افراد معتاد

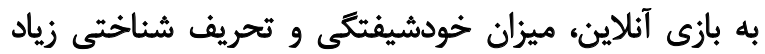

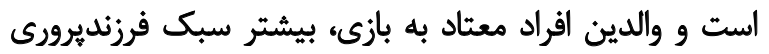

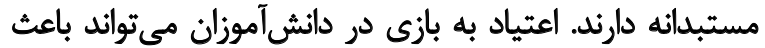

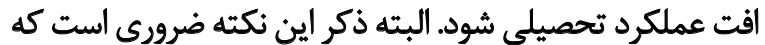

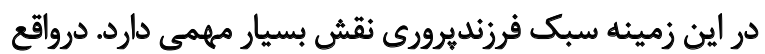

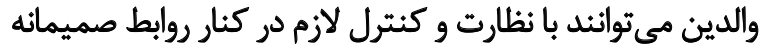

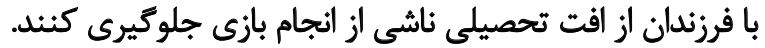

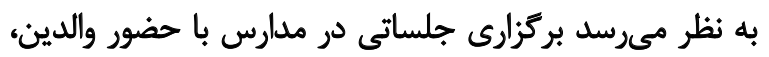

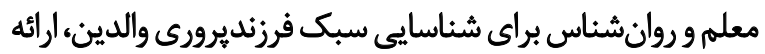

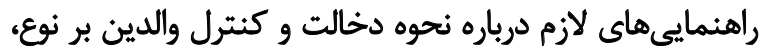
زمان و هكونكى بازىها بسيار موثر باشد. 


\section{References}

[1] Li H, Wang S. The role of cognitive distortion in online game addiction among Chinese adolescents. Children and Youth Services Review. 2013; 35(9):1468-75. [DOI:10.1016/j.childyouth.2013.05.021]

[2] Iranian Banking. [Addiction to digital games is a disease (Persian)] [Internet]. 2018 [Updated 2018 January 09]. Available From: http://bankdariirani.ir/fa/news/123534/

[3] Liu M, Peng W. Cognitive and psychological predictors of the negative outcomes associated with playing MMOGs (Massively Multiplayer Online Games). Computers in Human Behavior. 2009; 25(6):1306-11. [DOI:10.1016/j.chb.2009.06.002]

[4] Müller W, Koch A, Dickenhorst U, Beutel E, Duven E, Wölfling $\mathrm{K}$. Addressing the question of disorder-specific risk factors of internet addiction: A comparison of personality traits in patients with addictive behaviors and comorbid internet addiction. BioMed Research International. 2013; 2013(546342):1-7. [DOI:10.1155/2013/546342]

[5] Van der Aa N, Overbeek G, Engels R, Scholte R, Meerkerk G, Van den Eijnden J. Daily and compulsive internet use and wellbeing in adolescence: A diathesis-stress model based on big five personality traits. Journal of youth and adolescence. 2009; 38(6):765-76. [DOI:10.1007/s10964-008-9298-3] [PMID]

[6] Ko H, Yen Y, Yen F, Lin C, Yang J. Factors predictive for incidence and remission of internet addiction in young adolescents: A prospective study. CyberPsychology \& Behavior. 2007; 10(4):54551. [DOI:10.1089/cpb.2007.9992] [PMID]

[7] Griffiths MD, Meredith A. Video game addiction and its treatment. Journal of Contemporary Psychotherapy. 2009; 39(4):24753. [DOI:10.1007/s10879-009-9118-4]

[8] Baer S, Bogusz E, Green A. Stuck on screens: Patterns of computer and gaming station use in youth seen in a psychiatric clinic. Journal of the Canadian Academy of Child and Adolescent Psychiatry. 2011; 20(2):86-94. [PMID] [PMCID]

[9] King DL, Delfabbro PH. The cognitive psychopathology of Internet gaming disorder in adolescence. Journal of Abnormal Child Psychology. 2016; 44(8):1635-45. [DOI:10.1007/s10802-0160135-y] [PMID]

[10] Kim Y, Park K, Cho B. Prevalence of internet addiction and correlations with family factors among South Korean adolescents. Adolescence, 43(172):895-909. [PMID]

[11] Park S, Kim S, Roh S, Soh M, Lee S, Kim H, et al. The effects of a virtual reality treatment program for online gaming addiction. Computer Methods and Programs in Biomedicine. 2016; 129:99108. [DOI:10.1016/j.cmpb.2016.01.015] [PMCID]

[12] Sheikholeslami R, Borzo SH. [Child-rearing and responsibility among adolescents: The mediating role of identity processing styles (Persian)]. Psychological Methods and Models. 2016; 6(19):97-120.

[13] Rastegar F, Dousti Y. [Predicted internet addiction students based on their parenting style (Persian)]. Journal of Psychology \& Behavioral Studies. 2016; 4(4):115-21.

[14] Pan W, Yi Qun G, Min L. Research on relationship between tendency to computer game addiction and parental rearing styles in senior high school students. Chinese Journal of Clinical Psychology. 2006; 14(15):460-2.
[15] Zamani E, Abedini Y. [Structural model of effect of parenting styles and computer game addiction on academic male students performance (Persian)]. New Educational Approaches. 2014; 2(18):133-56.

[16] Ahmadi kh, Sedghi Jalal H. [Family and internet addiction in adolescents (Persian)]. Papre presented at: The $1^{\text {st }}$ National Congress of Family Psychology, A Step in Drawing the Desirable Family Model. 20 November 2014; Ahwaz, Iran.

[17] Zeini Hasanvand N, Javanmard K, Goodarzi M. [Validation of the narcissistic personality inventory-40 (Persian)]. Journal of Psychology. 2015; 19(1):102-18

[18] Zandi Payam A, Davoudi I, Mehrabi Zadeh M. [Validation of online game addiction inventory (Persian)]. Iranian Journal of Psychiatry and Clinical Psychology. 2016; 21(4):351-61.

[19] Ansari Shahidi M , Zamani N. [Compare the level of knowledge and quality of life in older women with a husband without a wife and Recent disengagement city of Hamadan (Persian)]. Quarterly Journal of Nursing Elderly. 2017; 3(2):76-88.

[20] Jozitehrani F. [Anticipation of depression and anxiety based on cognitive distortions in Iranian students residing in Dubai (Persian)] [MSc. thesis]. Shiraz: University of Shiraz; 2013.

[21] Shahamat F, Sabeti A, Rezvani S. [Relationship between parenting styles and early maladaptive schemas (Persian)]. Educational and Psychology. 2010; 11(2):239-54.

[22] Babapour Kheiroddin J, Esmaeili B, Gholamzadeh M, Mohammadpoor $\mathrm{W}$. [The relationship between parenting styles and identity styles in adolescent (Persian)]. Journal of Psychology. 2012; 16(2):161-70

[23] Rezapour R, Zakeri M, Ebrahimi L. [Prediction of narcissism, perception of social interactions and marital conflicts based on the use of social networks (Persian)]. Journal of Family Research. 2017; 13(2):197-214

[24] Zargar Y, Davoudi I, Ahmadi V, Mihreshkar S. [Reviewing the psychometric properties of the short form of narcissistic scale and its relation to gender and education (Persian)]. Journal of Psychoanalysis. 2010; 2(1):127-15.

[25] Kirisci L, Tarter E, Vanyukov M, Reynolds M, Habeych M. Relation between cognitive distortions and neuro behavior disinhibition on the development of substance use during adolescence and substance use disorder by young adulthood: A prospective study. Drug and Alcohol Dependence. 2004; 76(2):125-33. [DOI:10.1016/j.drugalcdep.2004.04.015] [PMID]

[26] Toneatto T. Cognitive therapy for problem gambling. Cognitive and Behavioral Practice. 2002; 9(3):191-9. [DOI:10.1016/ S1077-7229(02)80049-9]

[27] Kim M, Han H, Lee S, Renshaw F. Combined cognitive behavioral therapy and bupropion for the treatment of problematic on-line game play in adolescents with major depressive disorder. Computers in Human Behavior. 2012; 28(5):1954-9. [DOI:10.1016/j.chb.2012.05.015]

[28] Xina M ,Xingb J ,Pengfeia W ,Hourua L , Mengchenga W Honga Z. Online activities, prevalence of Internet addiction and risk factors related to family and school among adolescents in China. Addictive Behaviors Reports. 2018; 7:14-8. [DOI:10.1016/j. abrep.2017.10.003] [PMID] [PMCID]

[29] Gunuc S, Ayten D. The relationships between Turkish adolescents' Internet addiction, their perceived social support and fami- 
ly activities. Computers in Human Behavior. 2013; 29(6):2197-207. [DOI:10.1016/j.chb.2013.04.011]

[30] Wen Li, Eric L, Garland, Matthew O. Howard a family factors in internet addiction among Chinese youth: A review of Englishand Chinese-language studies. Computers in Human Behavior. 2014; 31:393-411. [DOI:10.1016/j.chb.2013.11.004]

[31] Wang W, Dongping L, Xian L, Yanhui W, Wenqiang S, Liyan $\mathrm{Z}$, et al. Parent-adolescent relationship and adolescent internet addiction: A moderated mediation model. Addictive Behaviors. 2018; 84:171-7. [DOI:10.1016/j.addbeh.2018.04.015] [PMCID]

[32] Tolooei SH. [A study of the relation of work shifts and parents childbearing styles with student's internet addiction Dubai (Persian)] [MA. thesis]. Tehran: Islamic Azad University, Central Tehran Branch; 2015.

[33] Niemz K, Griffiths M, Banyard P. Prevalence of pathological Internet use among university students and correlations with self-esteem, the General Health Questionnaire (GHQ), and dis inhibition. Cyberpsychology \& Behavior. 2005; 8(6):562-70. [DOI:10.1089/cpb.2005.8.562] [PMID]

[34] Ng BD, Wiemer-Hastings P. Addiction to the internet and online gaming. Cyberpsychology \& Behavior. 2005; 8(2):110-3. [DOI:10.1089/cpb.2005.8.110] [PMID]

[35] Song I, LaRose R, Eastin MS, Lin CA. Internet gratifications and internet addiction: On the uses and abuses of new media. Cyberpsychology \& Behavior. 2004; 7(4):384-94. [DOI:10.1089/ cpb.2004.7.384] [PMID]

[36] Casale S, Fioravanti G. Why narcissists are at risk for developing Facebook addiction: The need to be admired and the need to belong. Addictive Behaviors. 2018; 76:312-8. [DOI:10.1016/j.addbeh.2017.08.038] [PMID] 